\title{
Beware the node: the more we know, the less aggressive we are
}

\author{
Antonio Piñero
}

B reast cancer management has changed in the last three decades more than in the last three centuries. These changes have been possible due to new concepts in the knowledge of tumour biology and technological advances. At this moment therapeutic decisions are based not only in clinical or pathological staging but in immunohistochemical characteristics, and even molecular characteristics of the tumour must be taken in account. It is possible that in a near future, perhaps earlier than we expect, we could decide on the treatment using only biological characteristics of the tumour. However currently staging is still necessary to determine the treatment. The state of regional lymph nodes is one of the parameters we need to know the TNM staging, which is the most widely used classification. Classically, spreading of cancer to lymph nodes was based on the orderly progression model proposed by Halsted, and it was assessed by studying all the removed nodes after doing an axillary lymphadenectomy. This is a surgeon- and pathologist-dependent method: the number and state of isolated nodes depend on the surgical dissection and more or less thorough pathological study.

In the 1990s clinical application of sentinel lymph node biopsy (SLNB) was described and it has become a widely accepted tool for breast cancer staging. It allows a more exhaustive analysis of the isolated sentinel lymph node and the application of more sensitive techniques, such as immunohistochemical studies. A negative sentinel node avoids axillary lymphadenectomy but a positive sentinel lymph node implies it should be done to know the extension of axillary lymph node affectation. This new approach has led the American Joint Committee on Cancer to change the TNM staging system in its latest edition, including SLNB as a variable to be considered [1].

The detection of extra-axillary lymph drainages from the primary tumour is another advantage of using SLNB. In the specific case of breast cancer, drainage to internal

\footnotetext{
A. Piñero (局)

Department of General Surgery

Virgen de la Arrixaca University Hospital

Ctra. Madrid-Cartagena, $\mathrm{s} / \mathrm{n}$

El Palmar, Murcia, Spain

e-mail: antonio.pinero@carm.es
}

mammary lymph nodes is the second pattern of regional nodal drainage, after the axillary pathway, but others such as supraclavicular, infraclavicular, cervical and contralateral axilla must be considered. This possibility forces the study of these potential drainages when they occur, and if they are affected it implies a re-staging of the patient and perhaps a change in treatment. So, this technique enables better and more specific knowledge of the disease in each particular case, permitting tailored management. It also improves the quality of life in breast cancer patients by avoiding some frequent adverse events secondary to axillary lymphadenectomy, such as seroma and lymphoedema production.

Recent improvements in mammographic technology and increased use of screening campaigns have resulted in a decrease in the size of newly diagnosed breast cancers. This decreased tumour size is associated with less advanced stage disease and fewer positive lymph nodes. For this reason, when a sentinel lymph node is positive and lymphadenectomy is completed, more than half of the patients do not present any other additional metastasis focus. So, perhaps it can be concluded that axillary dissection could be avoided in a low-risk subset of sentinel lymph node-positive patients.

But, what does "a low-risk" mean? Are there any factors that allow us to predict lymph node affectation when we find a positive sentinel lymph node? If it occurs, we would be able to correctly stage the patient with a minimum associated morbidity.

Some recognised international centres have developed scores and nomograms to try to answer these questions and to allow an even more conservative management of these patients, either for breast cancer or other tumours, like prostate cancer and melanoma. In the specific case of breast cancer, we note the nomograms of the Sloan-Kettering Cancer Center and Stanford University in the USA, and Tenon Hospital and Cambridge University in Europe. All these nomograms and scores try to calculate the probability of non-sentinel lymph node affectation in case of positive SLNB. Their aim is to do it in a simple way, by using clinical and pathological parameters that are easy to obtain.

The nomogram from the Sloan-Kettering Cancer Center [2] is based on eight variables: tumour size, type and grade, lymphovascular invasion, multifocality, oestrogen receptor status, method of sentinel lymph node metastasis detection, 
number of positive sentinel lymph nodes and number of negative sentinel lymph nodes. The other three scores are simpler than this and they use only three parameters. The Tenon axilla scoring system [3] is based on the number of positive sentinel nodes divided by the total number of sentinel nodes removed, the presence or absence of macrometastasis in the sentinel node and the histological primary tumour size. The nomogram developed at Cambridge University [4] uses the grade, the largest detected size of sentinel lymph node metastasis and the proportion of involved sentinel lymph nodes among all of those removed. The nomogram from Stanford University [5] uses, at least, tumour size, status of lymphovascular invasion and the largest size of sentinel lymph node metastasis. In all these cases, the nomograms or scores try to predict affectation of non-sentinel lymph nodes and they have been tested or validated by several series [6-8].

In this issue of Clinical and Translational Oncology, Fougo et al. [9] discuss the possibility of using tumour-related variables rather than sentinel node-related variables to know if non-sentinel lymph nodes could be affected when there is a positive SLNB in breast cancer. Although they found that sentinel node metastasis size is significantly different between negative and positive non-sentinel nodes, it was not associated to non-sentinel node affectation in multivariate analysis. On the other hand, three of the four cited predictive systems use parameters related to characteristics of sentinel lymph node metastasis, such as size or presence or absence of macrometastasis.

Nevertheless, they agree with the previous nomograms that the parameters that could be useful to predict such af- fectation are the primary tumour size, the presence of multifocality and the presence of peritumoral lymph channel invasion. These are all data from the primary tumour. This coincidence with primary tumour variables is an important fact to be remarked. Most of them could be known before performing the SLNB, unlike sentinel lymph node-related factors, which require a biopsy, and if more sensitive and specific models are developed, it will be possible to calculate the odds of sentinel lymph node affectation, even without SLNB. Perhaps the integration of advances in molecular characterisation of primary tumour in these diagnostic predictive tools should be considered.

Although nomograms and scores could help to select patients in whom lymphadenectomy is not useful, these tools are not efficient enough to be applied in clinical practice yet. One important reason for observed variation in nomogram and score performance could be that there is to date no standardised methodology for pathologic analysis of sentinel lymph nodes. This can lead to different results between theoretically similar series. However, they are more objective than the clinicians' opinions and all of them can help to estimate the probability of non-sentinel lymph node metastasis, but there is no cutoff nomogram score that indicates the necessity of a lymphadenectomy. The decision-making process to do it should not be based on the nomogram score alone. Multiple factors, including comorbidities or implications for systemic therapy, among others, must be considered as well.

In this field, we can ask ourselves for how long we will need lymph node staging to guide systemic therapy and to prevent local recurrence... but this is another story.

\section{References}

1. American Joint Committee on Cancer (2002) AJCC Cancer Staging Manual, 6th Edn. Springer Verlag, New York, USA

2. Van Zee KJ, Manasseh DM, Bevilacqua JL et al (2003) A nomogram for predicting the likehood of additional noidal metastases in breast cancer patients with a positive sentinel node biopsy. Ann Surg Oncol 10:1140-1151. http://www.mskcc.org/nomograms

3. Barranger E, Coutant C, Flahault A et al (2005) An axilla scoring system to predict non-sentinel lymph node status in breast cancer patients with sentinel lymph node involvement. Breast Cancer Res Treat 91:113-119
4. Pal A, Provenzano E, Duffy SW et al (2008) A model for predicting non-sentinel lymph node metastatic disease when the sentinel lymph node is positive. Br J Surg 95:302-309

5. Kohrt HE, Olshen RA, Bermas HR et al (2008) New models and online calculator for predicting non-sentinel lymph node status in sentinel lymph node positive breast cancer patients. BMC Cancer 8:66-71. http://www-stat.stanford.edu/ olshen/ NSLNcalculator

6. Coutant C, Rouzier R, Fondrinier E et al (2008) Validation of the Tenon breast cancer score for predicting non-sentinel lymph node status in breast cancer patients with sentinel lymph node metastasis: a prospective multicenter study. Breast Cancer Res Treat 113:537-543
7. Poirier E, Sideris L, Dube P et al (2008) Analysis of clinical applicability of the Breast Cancer Nomogram for positive sentinel lymph node: the Canadian experience. Ann Surg Oncol 15:25622567

8. Gur AS, Unal B, Johnson R et al (2009) Predictive probability of four different breast cancer nomograms for nonsentinel axillary lymph node metastasis in positive sentinel node biopsy. J Am Coll Surg 208:229-235

9. Fougo JL, Afonso M, Senra FS et al (2009) Predictive factors for non-sentinel lymph node involvement in breast cancer patients with a positive sentinel node: should we consider sentinel node related factors? Clin Transl Oncol 11:165171 